\title{
A study about the improvement of Ant colony algorithm
}

\author{
Liang Wen-zhe ${ }^{1}$, Niu Qing-yin ${ }^{1}$, Fan Chao $^{2}$ \\ ${ }^{1}$ Department of Fundamental Courses, Academy of Armored Force Engineering, Beijing 100072, \\ China
}

${ }^{2}$ No. 900, Huangmei bridge, Huangmei town, Jurong City, Jiangsu Province 212421, China.

Keywords: ant colony algorithm; genetic algorithm; crossover operation

\begin{abstract}
The ant colony algorithm is a new kind of simulated evolutionary algorithm for random searching, which converges to the optimal path by the accumulation of pheromone and updating. But it lacks the global searching ability, and easily falls into local minimum and appears the phenomenon of premature stagnation. In this paper, the improved method, which applies a stronger global searching ability of genetic algorithm and introduces the improved crossover and mutation operation, is designed to enhance the global searching ability of ant colony algorithm. The improved algorithm is applied to a two-dimensional function optimization simulation calculation. The results show that the improved ant colony algorithm is better than the original in accuracy and time efficiency
\end{abstract}

\section{Introduction}

Ant colony algorithm is a kind of general bionic parallel algorithm, which was born in recent years. This new type of evolutionary algorithm is proposed by the Italy scholar Dorigo M. et al. in 1990s initially [1]. It imitates the foraging behavior of ants in the living world. An optimal solution could be obtained by updating the pheromone and converging to the optimal path continuously. The ant colony algorithm has strong robustness. If we have small changes on the modification, it still can be applied to other combinatorial optimization problems. It is also a positive feedback algorithm, which uses the feedback information of the system in a better way; and meanwhile, Ant colony algorithm is easy to combine with other heuristic algorithm to improve the performance of the algorithm. The algorithm lacks global search ability, so it is easy to fall into local minimum, and causes a premature stagnation phenomenon at result [2].

\section{Basic principles of ant colony algorithm}

In the process of searching optimization, according to the amount of information on each path and heuristic information element, the ant calculated the state transition probability. The formula is as follows [3][4]:

$$
p_{i j}^{k}(t)=\left\{\begin{array}{c}
\frac{\left[\tau_{i j}(t)\right]^{\alpha} \cdot\left[\eta_{i j}(t)\right]^{\beta}}{\sum_{\text {scallowed }_{k}}\left[\tau_{i s}(t)\right]^{\alpha} \cdot\left[\eta_{i s}(t)\right]^{\beta}}, j \in \text { allowed }_{k} \\
0, \text { otherwise }
\end{array}\right.
$$

In this formula, $p_{i j}^{k}(t)$ is the State transition probability of the ant $k$, when it moved from city $i$ to city $j$ at the time $t$. allowed ${ }_{k}=\left\{C-t a b u_{k}\right\}$ is the next city where the ant $k$ could move to. The tabu list $t a b u_{k}$ shows us the amount of city which the ant $k$ had passed. $p_{i j}^{k}(t)$ is proportional to $\tau_{i j}^{\alpha} \cdot \eta_{i j}^{\beta}, \eta_{i j}$ is visibility factor, $\alpha$ reflects the information of the ants in the movement process, and $\beta$ reflects the relative importance of heuristic information in the path of ants' choice. Too much residual information will cause the loss of heuristic information. As a result, the residual information should be updated after the ant finished one step or completed a cycle of all nodes. Therefore, at the time $t+n$, the formula is as follows: 


$$
\begin{aligned}
& \tau_{i j}(t+n)=(1-\rho) \cdot \tau_{i j}(t)+\Delta \tau_{i j}(t) \\
& \Delta \tau_{i j}(t)=\sum_{k=1}^{m} \Delta \tau_{i j}^{k}(t)
\end{aligned}
$$

$\rho$ indicates that the pheromone evaporation coefficient, In order to prevent the accumulation of the pheromone, the range of $\rho$ should be $[0,1] . \Delta \tau_{i j}(t)$ means the increasing of the route $(i, j)$ in one cycle at the time $t$. The initial time $\Delta \boldsymbol{\tau}_{\boldsymbol{i j}}$ is 0 . The steps of ant colony algorithm are:

1) Initialization. The maximum number of cycles is $N_{C \max }, \tau_{i j}(0)=B, \Delta \tau_{i j}(0)=0, \mathrm{~B}$ is a constant.

2) The cycle times is describe as $N_{c}=N_{c}+1$, and $k=1$ in $t a b u_{k}$.

3) The ant moves to the next city on the basis of formula (1).

4) Record the city where the ant had arrived at. In $\operatorname{tabu}_{k}, \mathrm{k}=\mathrm{k}+1$.

5) If $\mathrm{k}<\mathrm{m}$ (the number of the city), it should turn back to step 3), otherwise turn to step 6).

6) According to the formula (2) and (3), the local information of each path should be updated.

7) If Cycle times is showed as $N_{c} \geq N_{C \max }$, the cycle finish and calculation results should be output, otherwise the cycle will keep on.

\section{Improvement of ant colony algorithm}

It shows at previous that ant colony algorithm mainly relies on positive feedback mechanism. But it is easy to fall into local optimal solution, appeared with stagnation phenomenon. Inspired by the usage of genetic algorithms in Qin Ling's article[5], the author proposes an improved scheme which is based on the crossover and mutation operation in genetic algorithm. In order to increase the population diversity of ant colony algorithm, the randomness is added to improve the crossover and the global search ability. In this conception, the ant colony algorithm will not be transferred to the next immediately, it waits until the crossover and mutation operation have finished.

Crossover is used to combination of new individual. It imitates the biological hybridization, which produces a new individual by crossing transposition of partial genes on different chromosomes. The performance of genetic algorithm is largely determined by the cross operation.

For a better application in ant colony algorithm, in this paper, the implementation strategy of the crossover has been changed. In the traditional strategy, the process of crossover is happened between two randomly selected ants. But if the ants is not the most optimal on at the updating, a large number of invalid searches will be produced. In this paper, the strategy is designed as: when iteration finished, an optimal ant will be chosen to get cross with one random selected and or one group of ants. If the new individual is better than the current optimal one, then it will take the place of the old one as optimal ant, otherwise it will be rejected as the new individual.

According to the improved strategy designed in this paper, the algorithm of the improved ant colony algorithm is as follows:

1) Initialization. sets $m$ as the number of the ant.

2) According to the state transfer probability formula, each ant chooses the next city. After completing a traversal, the local pheromone in the adjacent nodes will be updated.

3) Select the current optimal ant.

4) Crossover the optimal one with one or a group of random selected ants.

5) Select the better one as the new optimal ant.

6) Select the better one as the new optimal ant, after the mutation operation of ant colony by mutation probability.

7) Update the global pheromone, if $N_{c} \geq N_{C \max }$, stop the cycle and output calculation results, otherwise go back to step 2).

The procedure of ant colony algorithm is describes in Fig 1: 


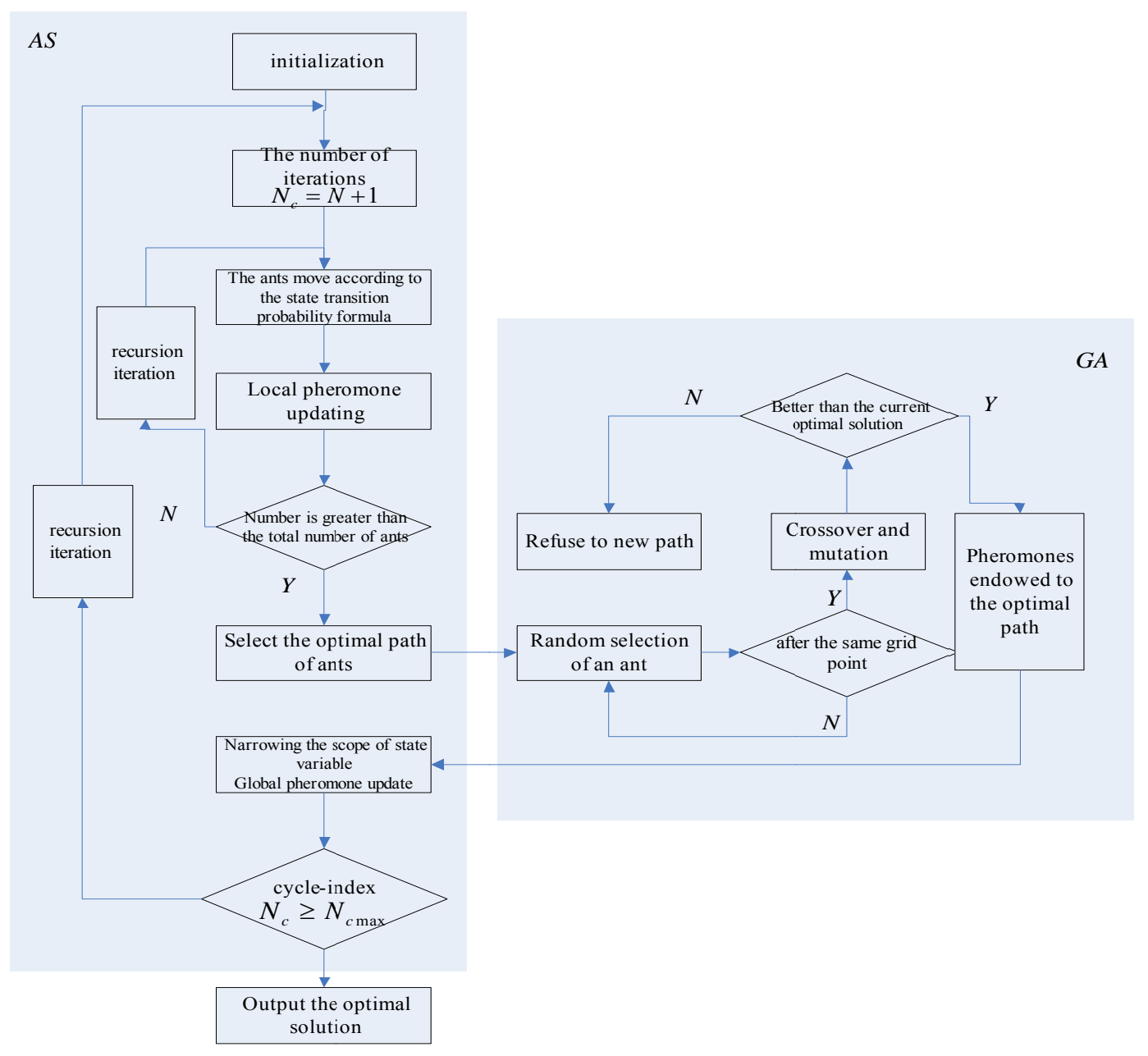

Fig 1 The procedure of ant colony algorithm

\section{The optimization application of improved ant colony algorithm}

In order to verify the performance of the improved ant colony algorithm, the paper introduces a two dimensional function. $\min F=\left(1-x_{1}\right)^{2}+10\left(x_{1}-x_{2}\right)^{2}$ with $x_{i} \in[-1,1], i=1,2$. It is easy to get that if $x_{i}$ equals $1(i=1,2)$, we have $\min F=0$. Compared with origin, take $\mathbf{B}=\mathbf{0 . 0 1} \mathrm{m}=500$ $\alpha=1 \quad \beta=1 \quad \rho=0.99 Q=10$ (the crossover and mutation operation) $\varepsilon=0.001$. We get shows the simulation result in Fig 2.

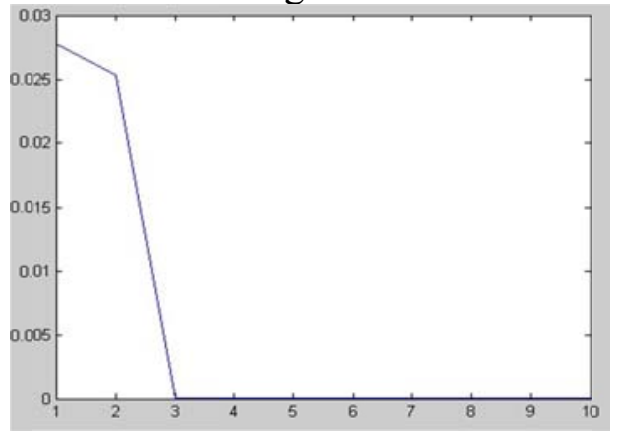

(a)ant colony algorithm without improved

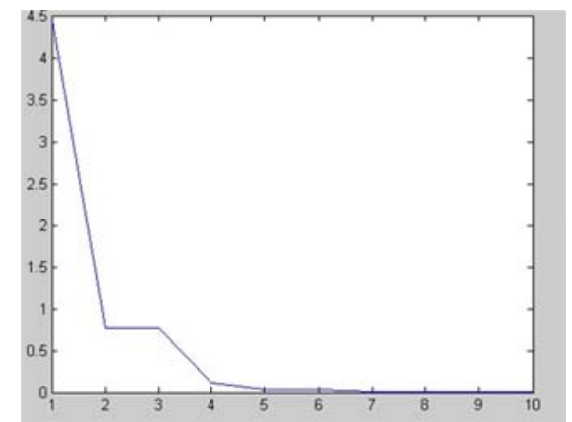

(b) improved ant colony algorithm

Fig 2 The simulation results of ant colony algorithm without improved and improved ant colony algorithm 
Table 1 Comparison of algorithm before and after improvement

\begin{tabular}{|c|c|c|}
\hline Ant colony optimization algorithm & Optimal value & Optimal solution $\left(x_{1}, x_{2}\right)$ \\
\hline Before & $1.29 \mathrm{e}-003$ & $(0.9766,1.0118)$ \\
\hline After & $4.1000 \mathrm{e}-007$ & $(1.0001,1.0003)$ \\
\hline
\end{tabular}

In table 1 , it shows that the improved ant colony algorithm could obtain more accurate solution than the original one in solving the two dimensional optimization problems, and it proves that the improved ant colony algorithm has good performance and feasibility.

\section{Conclusion}

Ant colony algorithm is a new optimization algorithm with a lot of advantages. But the lack of global search ability has restricted its application and development. In this paper, the author introduced the genetic algorithm which has stronger global search ability into ant colony algorithm, and we got a good result that this improvement strategy is feasible and effective.

\section{References}

[1] Yang Jian-feng. Research on ant colony algorithm and its application [D]. Doctoral Dissertation of Zhejiang University.2007

[2] He Gui-liang,Pan Jiu-hui. Small improvement of ant colony algorithm [J]. Modern computer.2005

[3] M. Dorigo, V. Maniezzo, and A. Colorni. Ant system: optimization by a colony of cooperating agents. IEEE Transactions on Systems, Man, and Cybernetics-Part B, 1996, 26(1): 29 41

[4] M. Dorigo, L M. Gambardella. Ant colony system: a cooperative learning approach to the traveling salesman problem. IEEE Transactions on Evolutionary Computation, 1997, 1(1): 53 66

[5] Qin Ling,The Improvement and Application of The Ant Colony Algorithm, Yangzhou: Master degree thesis of Yangzhou University,2004. 